\title{
Expressions of the $\gamma_{2}$ chain of laminin-5 and secreted protein acidic and rich in cysteine in esophageal squamous cell carcinoma and their relation to prognosis
}

\author{
Li-Yan Xue, Shuang-Mei Zou, Shan Zheng, Xiu-Yun Liu, Peng Wen, Yan-Ling Yuan, Dong-Mei Lin, \\ and Ning Lu
}

Abstract

Previous studies have shown that the expressions of the $\gamma_{2}$ chain of laminin-5 and secreted protein acidic and rich in cysteine (SPARC) play important roles in oncogenesis and the development of carcinoma. To assess the expressions of laminin-5 $\gamma_{2}$ chain and SPARC in esophageal squamous cell carcinoma (SCC), and to clarify the prognostic significance of the expressions of laminin-5 $\gamma_{2}$ chain and SPARC in esophageal SCC, we detected the expressions of laminin-5 $\gamma_{2}$ chain and SPARC in cancer tissue and corresponding normal mucosa from 116 patients with advanced (stages II-IV) esophageal SCC using the tissue microarray-based immunohistochemistry and analyzed the correlation of the expressions with clinicopathologic characteristics and survival. We found that in normal esophageal tissues, laminin-5 $\gamma_{2}$ chain was expressed in the basement membrane, whereas in esophageal SCC tissues, laminin-5 $\gamma_{2}$ chain was expressed in the cytoplasm of carcinoma cells, with a positive rate of $72.4 \%$. SPARC was not detected in normal esophageal mucosa, but was expressed in stromal fibroblasts in $84.6 \%$ of esophageal SCC cases and in cancer cells in $7.8 \%$ of esophageal SCC cases. There was a significant correlation between laminin-5 $\gamma_{2}$ chain and stromal SPARC expression in esophageal SCC (Spearman's rho $=0.423$, $P<0.001)$. The expressions of both laminin-5 $\gamma_{2}$ chain and stromal SPARC were correlated with survival ( $P=0.032$ and $P=0.034$, respectively). In stage-II esophageal SCC, the expression of laminin-5 $\gamma_{2}$ chain was significantly correlated with survival $(P=0.023)$, while the expression of SPARC was not significantly correlated with survival $(P=0.154)$. Patients with elevated levels of laminin-5 $\gamma_{2}$ chain and SPARC expressions had a poorer prognosis than did those lacking elevated levels of laminin- $5 \gamma_{2}$ chain expression and/or elevated levels of SPARC expression $(P=0.001)$. In stage-II esophageal SCC, patients with elevated levels of laminin- $5 \gamma_{2}$ chain and SPARC expressions had a poorer prognosis $(P<0.001)$. These results suggest that laminin- $5 \gamma_{2}$ chain and SPARC may play roles in the progression of esophageal SCC and their simultaneous expression is correlated with poorer prognosis, especially in patients with stage-II SCC.

Key words Laminin-5 $\gamma_{2}$, SPARC, esophageal cancer, squamous cell carcinoma, pathology

Esophageal squamous cell carcinoma (SCC) is a

\footnotetext{
Authors' Affiliation: Department of Pathology, Cancer Institute/Hospital, Peking Union Medical College, Chinese Academy of Medical Sciences, Beijing 100021, P. R. China.

Corresponding Author: Ning Lu, Department of Pathology, Cancer Institute/Hospital, Peking Union Medical College, Chinese Academy of Medical Sciences, Beijing 100021, P. R. China. Tel: +86-10-87788435; Email: nlu03@126.com.
}

common malignant tumor in the digestive tract and its incidence among the Chinese population ranks first in the world. The prognosis of esophageal SCC is very poor, mainly because severe invasion and lymph node metastases have already occurred by the time the disease is diagnosed. Thus, most patients being treated for esophageal SCC are at an advanced stage. Strikingly, the 5-year survival rate of patients with early stage esophageal SCC is up to $80 \%-90 \%$, whereas that of patients with advanced stage esophageal SCC is less 
than $30 \%$. Thus, it is of great significance to explore selective biomarkers that are able to predict the prognosis of esophageal SCC and further guide clinical treatment.

Cancer invasion and metastasis require degradation of the extracellular matrix and connective tissues surrounding the tumor. Laminins are a family of extracellular matrix proteins localized at the basement membrane and are involved in cell adhesion, migration, proliferation, and differentiation. Laminin- 5 belongs to the laminin family and is a hetero-trimeric glycoprotein composed of $\alpha_{3}, \beta_{3}$, and $\gamma_{2}$ subunits. The $\gamma_{2}$ chain is unique to laminin $-5^{[1]}$. The secreted protein acidic and rich in cysteine (SPARC) is one of the non-structural matricellular proteins. SPARC mediates the interaction of the matrix with the cell, but does not participate in the structural formation of the extracellular matrix ${ }^{[2]}$. Whether they are involved in extracellular matrix formation and stabilization differentiates extracellular matrix proteins such as laminin-5 $\gamma_{2}$ from matricellular proteins such as SPARC. Both laminin-5 $\gamma_{2}$ chain and SPARC are overexpressed in many types of cancers and play important roles in tumorigenesis and cancer progression. Therefore, these proteins are of clinical significance to predict the prognosis of advanced cancers. It has been reported that the expression levels of laminin-5 $\gamma_{2}$ chain are significantly higher in gastric cancer, head and neck cancer, tongue cancer, colon cancer, breast cancer, cervical cancer, and skin cancer than in corresponding normal tissues and that the overexpression of laminin-5 $\gamma_{2}$ chain is predominantly observed in the cytoplasm of carcinoma cells at the invasive front. The expression and function of SPARC in human cancer tissues is more complicated and depends on cancer type and cellular microenvironment. SPARC is highly expressed in breast cancer, colon cancer, gastric cancer, bladder cancer, liver cancer, and prostate cancer. In addition to cancer cells, stromal cells, usually fibroblasts, also express SPARC. Although the expressions of laminin-5 $\gamma_{2}$ chain and SPARC have been extensively studied in the above-mentioned cancers ${ }^{[3-8]}$, their expressions in advanced esophageal SCC and its significance in prognosis are still unclear. Yamamoto et al. ${ }^{\left[{ }^{9]}\right.}$ reported the expression of laminin-5 $\gamma_{2}$ chain in esophageal cancer is related to the age, gender, invasion degree (only a trend without statistical difference), lymph node metastasis, and pTNM stage. In addition, the high expression of laminin-5 $\gamma_{2}$ chain appeared to be linked with poor prognosis. Yamashita et al. ${ }^{[10]}$ demonstrated the localization of SPARC protein in both cancer cells and stromal cells of esophageal cancer using immunohistochemistry. However, they didn't do statistical analysis of the relation between SPARC protein expression and prognosis, owing to a small number of patients. Instead, Northern blot hybridization suggested the expression of SPARC mRNA is related to lymph node metastasis and poor prognosis. With such a paucity of findings, the expression and role of both laminin-5 $\gamma_{2}$ chain and SPARC in advanced esophageal SCC warrant further investigation.

The laminin- $5 \gamma_{2}$ chain has been reported degraded by matrix metalloproteinase (MMP) secreted from cancer cells or adjacent stromal cells, resulting in an increased capacity of migration and/or invasion in cancer cells. Thus, laminin-5 $\gamma_{2}$ chain may serve as an effective target during cell migration and invasion ${ }^{[11]}$. Overexpression of laminin-5 $\gamma_{2}$ chain increases cell proliferation, and thereby promotes tumor growth ${ }^{[12]}$. In contrast, SPARC increases the expression of MMP in fibroblasts and monocytes. Since MMP enhances tumor cell degradation of the extracellular matrix, its overexpression enhances cell movement and is strongly related to cancer invasion, metastasis and prognosis ${ }^{[13-18]}$. Therefore, it is intriguing to suppose that laminin-5 $\gamma_{2}$ chain and SPARC have a synergistic effect in tumor progression and metastasis. Moreover, SPARC has been shown to interact directly with laminin, and its expression is related to laminin production ${ }^{[19-21]}$. It is possible that SPARC potentiates cancer progression and metastasis through its ability to enhance laminin expression. However, the relationship between the $\gamma_{2}$ chain of laminin and SPARC has not yet been fully studied.

The present study aimed to detect the expressions of laminin-5 $\gamma_{2}$ chain and SPARC in advanced esophageal SCC using tissue microarray based on immunohistochemistry and to determine their correlation with clinicopathologic characteristics and survival of patients. In addition, we also investigated the relationship between the expressions of laminin-5 $\gamma_{2}$ chain and SPARC to clarify their synergic effect on the progression of esophageal SCC.

\section{Patients and Methods}

\section{Patients and sample collection}

Specimens were collected from 116 cases of esophageal SCC. All the patients had undergone curative surgical resection at Cancer Hospital, Peking Union Medical College, Chinese Academy of Medical Sciences between June 2001 and June 2002. No preoperative radiotherapy or chemotherapy was performed for all of these patients. Of the 116 patients, 94 were male and 22 were female, aged ranging from 33 to 78 years, with a median age of 59.5. The carcinoma tissue and the corresponding normal mucosa were fixed with $80 \%$ ethanol and embedded in paraffin. Of the 116 esophageal SCC, 31 were well differentiated, 56 were moderately differentiated, and 29 were poorly 
differentiated. The clinicopathologic characteristics of all patients were evaluated according to the guidelines of International Union Against Cancer (UICC) 2002 pTNM criteria. Of the 116 cases, 43 were at stage IIA, 11 were at stage IIB, 53 were at stage III, and 9 cases were at stage IV.

\section{Follow-up}

Survival time was defined as the time from surgery to death or the last follow-up visit. Patients dying of serious complications during surgery, of causes other than esophageal SCC, or in less than 3 months after surgery were not included. The longest survival time was 89 months by the last follow-up visit, and the median survival time is 36 months.

\section{Construction of tissue microarray}

Representative areas containing morphologically representative SCC and regions of normal mucosa were constructed on donor paraffin blocks based on hematoxylin and eosin (HE) staining. The appropriate tissues were constructed on receptor paraffin blocks using a tissue microarrayer (Beecher Instruments, Silver Spring, MD, $0.6 \mathrm{~mm}$ in diameter of cores) ${ }^{[22,23]}$. Each case of SCC and corresponding normal mucosa were loaded twice to ensure representation of the chip and to avoid missing information due to loss of tissue cores.

\section{Immunohistochemistry assay and data analysis}

A standard streptavidin-peroxidase conjugated method (SP) was used for immunohistochemistry assay. Slides were de-waxed in xylene and hydrated through gradient ethanol to distilled water. Subsequently, antigen retrieval was performed as follows. For laminin-5 $\gamma_{2}$ chain, slides were incubated with protease XXIV (Biogenex, USA) at room temperature for $10 \mathrm{~min}$. For SPARC, slides were microwaved in $0.01 \mathrm{~mol} / \mathrm{L}$ citrate buffer $(\mathrm{pH} \mathrm{6.0)}$ for $10 \mathrm{~min}$. Slides were then incubated with $3 \% \quad \mathrm{H}_{2} \mathrm{O}_{2}$ for $10 \mathrm{~min}$ to block endogenous peroxidase, blocked in $10 \%$ normal goat serum for 10 $\mathrm{min}$ to reduce non-specific staining and incubated with 1:50 dilutions of primary antibodies against laminin-5 $\gamma_{2}$ chain (mouse monoclonal antibody, Chemicon, USA) and against SPARC (mouse monoclonal antibody, Novacastra, UK) at room temperature for $1 \mathrm{~h}$. Slides were washed three times with PBS and incubated with biotinylated anti-mouse secondary antibodies for $10 \mathrm{~min}$ at room temperature followed by incubation with horseradish peroxidase streptavidin at room temperature for $10 \mathrm{~min}$. After DAB staining, slides were counter-stained with hematoxylin. PBS was used to replace primary antibodies as a negative control.
Scoring criteria for expression of laminin-5 $\gamma_{2}$ chain is as follows: negative (-), no laminin-5 $\gamma_{2}$ positive cells; weakly positive $(+)$, less than $30 \%$ cells are laminin-5 $\gamma_{2}$ positive; and strongly positive $(++)$, more than $30 \%$ of cells are laminin-5 $\gamma_{2}$ positive ${ }^{[24]}$. Different scoring criteria for SPARC were used for stromal cells and cancer cells/normal cells. Scoring criteria for stromal cells is as follows: negative (-), no fibroblasts are SPARC positive; weakly positive $(+)$, less than $50 \%$ of fibroblasts are SPARC positive; and strongly positive $(++)$, more than $50 \%$ of fibroblasts are SPARC positive ${ }^{[2]}$. Scoring criteria for cancer/normal epithelial cells is as follows: negative $(-)$, no cells are SPARC positive; weakly positive $(+)$, SPARC positive cells are less than $30 \%$; and strongly positive $(++)$, SPARC positive cells are more than $30 \%$. The intensity of immunostaining is comparable within all of the cases for both proteins; however, the percentage of immuno-positive cells is significantly different. Therefore, we only considered the percentage of immuno-positive cells for data analysis.

\section{Groupings}

To determine the role of laminin-5 $\gamma_{2}$ chain and SPARC in the progression of esophageal SCC, we divided 116 cases into two groups. Group A includes cases at stages IIA and IIB, while group B includes cases at stages III and IV. To determine the significance of elevated co-expression of laminin-5 $\gamma_{2}$ chain and SPARC, we divided the 116 cases into three groups: in group one, neither laminin-5 $\boldsymbol{\gamma}_{2}$ chain nor SPARC is highly expressed; in group two, either laminin-5 $\gamma_{2}$ chain or SPARC is highly expressed; and in group three, both laminin-5 $\gamma_{2}$ chain and SPARC are highly expressed. Group $A$ is further categorized into three subgroups: group A1 consists of cases in which neither laminin-5 $\gamma_{2}$ chain nor SPARC highly expressed, group A2 consists of cases in which either laminin-5 $\boldsymbol{\gamma}_{2}$ chain or SPARC highly expressed, and group A3 consists of cases in which both laminin-5 $\gamma_{2}$ chain and SPARC highly expressed.

\section{Statistical analysis}

SPSS16.0 statistical software was used for statistical analysis. The $\chi^{2}$ test or Fisher's exact test was utilized to analyze the relationship between laminin-5 $\gamma_{2}$ chain and SPARC expressions in cancer tissue and the clinicopathological characteristics. Spearman rank correlation coefficient was calculated to determine the correlation between these two proteins. Furthermore, the Kaplan-Meier survival analysis with log-rank test was performed to analyze the relationship between the expression levels of the two proteins in cancer tissue or 
other clinicopathologic characteristics and the survival rate of patients. A value of $P<0.05$ was defined as significantly different.

\section{Results}

\section{Expressions of laminin-5 $\gamma_{2}$ chain and SPARC in SCC and normal mucosa}

In squamous cells of normal esophageal mucosa, laminin-5 $\gamma_{2}$ was localized at the basement membrane but not in the cytoplasm. In contrast, laminin-5 $\gamma_{2}$ was expressed in the cytoplasm of cancer cells, predominating in cells at the invasive front of the tumor. Of the 116 cases, 66 (56.9\%) were weakly positive and $18(15.5 \%)$ were strongly positive for laminin-5 $\gamma_{2}$ expression. SPARC was not expressed in normal esophageal epithelium or stromal cells in lamina propria, but was strongly expressed in SCC stromal fibroblasts. Six cases had tumor nests without any visible stromal cells in the tissue microarray. Of the remaining 110 cases that contained stromal cells, 32 (29.1\%) were weakly positive and $61(55.5 \%)$ were strongly positive. Only 9 cases $(7.8 \%)$ showed SPARC positive in the cytoplasm of cancer cells at the invasive front of tumor nests, which included 4 weakly positive cases and 5 strongly positive cases (Figure 1).

The relationship between the expression levels of laminin-5 $\gamma_{2}$ chain and SPARC and clinicopathologic characteristics of SCC

Laminin-5 $\gamma_{2}$ expression was associated with the degree of cancer differentiation, being highly expressed in moderately differentiated and poorly differentiated carcinomas $(P=0.004)$ (Table 1$)$. However, SPARC expression in stromal cells and cancer cells did not exhibit any correlation with clinicopathologic characteristics $(P>0.05)$ (Table 2$)$.

The relationship between laminin-5 $\gamma_{2}$ chain and SPARC expression

The expression of laminin-5 $\gamma_{2}$ chain increased as the expression of SPARC in stromal fibroblasts increased (Spearman correlation coefficient is $0.423, P<$ 0.001). However, the expression level of laminin-5 $\gamma_{2}$ chain was not associated with SPARC expression level in cancer cells (Spearman correlation coefficient is $-0.100, P=0.287$ ) (Table 3).

\section{Overall survival analysis}

The 5-year survival rate of the 116 patients in this study was $41.7 \%$. The Kaplan-Meier survival analysis showed that age, lymph node metastasis, invasive depth, pTNM stage, the expression levels of laminin-5 $\gamma_{2}$ chain and SPARC in stromal cells were related to the survival rate $(P=0.002, P=0.002, P<0.001, P<$ $0.001, P=0.032$, and $P=0.034$, respectively). As shown in Figure 2A-D, the 5-year survival rate of patients with strongly positive laminin-5 $\gamma_{2}$ chain expression was $22.2 \%$, whereas that of patients with negative and weakly positive laminin-5 $\gamma_{2}$ expression was $45.5 \%$. The 5-year survival rate of patients with strongly positive SPARC expression in stromal cells was $32.7 \%$, whereas that of patients with negative and weakly positive SPARC expression was $52.0 \%$. The 5 -year survival rate of patients with positive SPARC expression in cancer cells was $37.5 \%$, while that of patients with negative SPARC expression was $42 \%(P=0.944)$.

\section{Survival analysis of group A and group B}

For the patients within group A (stage IIA/IIB), the expression level of laminin-5 $\gamma_{2}$ chain was significantly correlated with the survival rate $(P=0.023)$. The 5-year survival rate of patients with strong expression was $33.3 \%$, while that of patients with negative and weakly positive expression of laminin-5 $\gamma_{2}$ chain was $72.1 \%$. For patients in group B (stage III/IV), the expression level of laminin-5 $\gamma_{2}$ chain was not significantly related to the survival rate $(P=0.844)$. The 5 -year survival rate of patients with strongly positive laminin-5 $\gamma_{2}$ chain expression was $16.7 \%$, while that of patients with negative and weakly positive expression was $17.8 \%$. In group A, the 5-year survival rate of patients with strongly positive SPARC expression in stromal cells was $56.7 \%$, which was not statistically different from that of patients with negative and weakly positive SPARC expression $(75.0 \%)(P=0.154)$. In group $B$, the 5-year survival rate of patients with strongly positive SPARC expression in stromal cells was $18.9 \%$, while that of patients with negative and weakly positive SPARC expression was $16.7 \% \quad(P=0.988)$. The survival rate of patients with positive SPARC expression in cancer cells was not investigated owing to the paucity of cases (Figure $2 \mathrm{E}-\mathrm{H}$ ).

Survival analysis of co-expression laminin-5 $\gamma_{2}$ chain and SPARC

Patients were divided into 3 groups according to the expression of laminin-5 $\gamma_{2}$ chain and SPARC as described in the method section. Patients in group three, with a strong expression of laminin-5 $\gamma_{2}$ chain and SPARC, had a 5-year survival rate of $7.7 \%$, while patients in group one, without strong expression of laminin- $5 \gamma_{2}$ chain or SPARC, had a 5-year survival rate of $51.0 \%$ and patients in group two, with strong expression of 


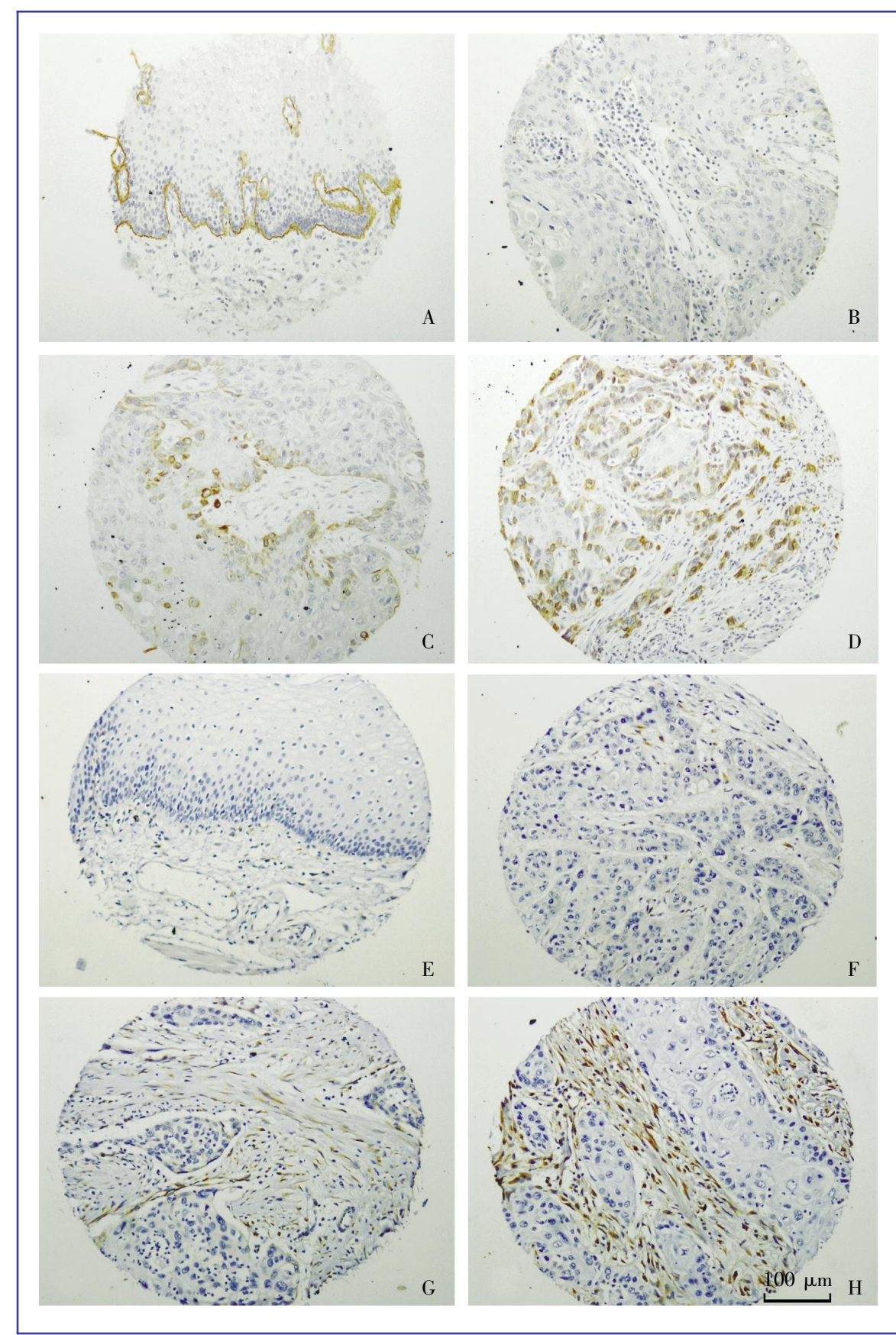

Figure 1. Immunohistochemistry for laminin-5 $\gamma_{2}$ chain and SPARC in esophageal squamous cell carcinoma (SCC). A, laminin-5 $\gamma_{2}$ chain is strongly expressed in the basement membrane of normal esophageal epithelia. B, laminin-5 $\gamma_{2}$ chain was not detected in esophageal SCC. C, laminin-5 $\gamma_{2}$ chain is weakly expressed in the cytoplasm of esophageal SCC, mainly at the tumor-stroma interface. D, laminin-5 $\gamma_{2}$ chain is strongly expressed in the cytoplasm of esophageal SCC, mainly at the tumor-stroma interface. E, SPARC was not detected in the epithelia or lamina propria mucosa stroma of normal mucosa. F, SPARC was not detected in the cancer cells or stromal fibroblasts in esophageal SCC, with expression only exhibiting in the endothelia of small vessels. G, SPARC is weakly expressed in stromal fibroblasts in esophageal SCC but was not detected in cancer cells. H, SPARC is strongly expressed in stromal fibroblasts in esophageal SCC but was not detected in cancer cells. Bar, $100 \mu \mathrm{m}$.

either laminin-5 $\gamma_{2}$ chain or SPARC, had a 5-year survival rate of $41.9 \%$. There was significant difference among these three groups $(P=0.001)$, as shown in Figure 2I. In patients with stage-IIA/IIB esophageal SCC, the 5-year survival rate of the A3 subgroup, which strongly expressed laminin-5 $\gamma_{2}$ chain and SPARC, was 0 , while the 5 -year survival rate of the A1 subgroup and the A2 subgroup was $73.1 \%$ and $73.3 \%$, respectively. The 5-year survival rates of the three subgroups were significantly different $(P<0.001)$, as shown in Figure $2 \mathrm{~J}$.

\section{Discussion}

Laminins are a family of extracellular matrix proteins localized at the basement membrane and are involved in cell adhesion, migration, proliferation, and differentiation. 
Table 1. Correlation between laminin-5 $\gamma_{2}$ chain expression and clinicopathologic characteristics in 116 patients with esophageal squamous cell carcinoma

\begin{tabular}{|c|c|c|c|c|c|}
\hline \multirow{2}{*}{ Clinicopathologic characteristic } & \multirow{2}{*}{ No. of patients } & \multicolumn{3}{|c|}{ Laminin $5 \gamma_{2}$ expression $(n=116)$} & \multirow{2}{*}{$P^{a}$} \\
\hline & & - & + & ++ & \\
\hline Age (years) & & & & & 0.745 \\
\hline$\geqslant 60$ & 58 & $15(25.9)$ & $35(60.3)$ & $8(13.8)$ & \\
\hline$<60$ & 58 & $17(29.3)$ & $31(53.4)$ & $10(17.2)$ & \\
\hline Gender & & & & & 0.296 \\
\hline Male & 94 & $23(24.5)$ & $56(59.6)$ & $15(16.0)$ & \\
\hline Female & 22 & $9(40.9)$ & $10(45.5)$ & $3(13.6)$ & \\
\hline Differentiation & & & & & 0.004 \\
\hline Well & 31 & $8(25.8)$ & $21(67.7)$ & $2(6.5)$ & \\
\hline Moderate & 56 & $9(16.1)$ & $35(62.5)$ & $12(21.4)$ & \\
\hline Poor & 29 & $15(51.7)$ & $10(34.5)$ & $4(13.8)$ & \\
\hline Invasive depth $(\mathrm{T})$ & & & & & 0.592 \\
\hline $\mathrm{T} 1 / 2$ & 22 & $8(36.4)$ & $11(50.0)$ & $3(13.6)$ & \\
\hline $\mathrm{T} 3 / 4$ & 94 & $24(25.5)$ & $55(58.5)$ & $15(16.0)$ & \\
\hline Lymph node metastasis (N) & & & & & 0.386 \\
\hline NO & 49 & $15(30.6)$ & $29(59.2)$ & $5(10.2)$ & \\
\hline N1 & 67 & $17(25.4)$ & $37(55.2)$ & $13(19.4)$ & \\
\hline pTNM stage & & & & & 0.286 \\
\hline$\| A / I B$ & 54 & 18(33.3) & $30(55.6)$ & $6(11.1)$ & \\
\hline III/IV & 62 & $14(22.6)$ & $36(58.1)$ & $12(19.4)$ & \\
\hline
\end{tabular}

Table 2. Correlation between SPARC expression and clinicopathologic characteristics in 116 esophageal esophageal squamous cell carcinoma patients

\begin{tabular}{|c|c|c|c|c|c|c|c|}
\hline \multirow{2}{*}{$\begin{array}{l}\text { Clinicopathologic } \\
\text { characteristic }\end{array}$} & \multicolumn{3}{|c|}{ SPARC expression in stromal fibroblasts $(n=110)^{a}$} & \multirow{2}{*}{$P^{\mathrm{b}}$} & \multicolumn{2}{|c|}{ SPARC expression in cancer cells $(n=116)$} & \multirow{2}{*}{$P^{c}$} \\
\hline & - & + & ++ & & - & $+/++$ & \\
\hline Age (years) & & & & 0.682 & & & 0.162 \\
\hline$\geqslant 60$ & $7(13.0)$ & $15(27.8)$ & $32(59.3)$ & & $51(87.9)$ & $7(12.1)$ & \\
\hline$<60$ & $10(17.9)$ & $17(30.4)$ & $29(51.8)$ & & $56(96.6)$ & $2(3.4)$ & \\
\hline Gender & & & & 0.191 & & & 1.000 \\
\hline Male & $12(13.5)$ & $24(27.0)$ & $53(59.6)$ & & $86(91.5)$ & $8(8.5)$ & \\
\hline Female & $5(23.8)$ & $8(38.1)$ & $8(38.1)$ & & $21(95.5)$ & $1(4.5)$ & \\
\hline Differentiation & & & & 0.128 & & & 0.368 \\
\hline Well & $2(6.9)$ & $8(27.6)$ & $19(65.5)$ & & $29(93.5)$ & $2(6.5)$ & \\
\hline Moderate & $8(15.1)$ & $13(24.5)$ & $32(60.4)$ & & $53(94.6)$ & $3(5.4)$ & \\
\hline Poor & $7(25.0)$ & 11(39.3) & $10(35.7)$ & & $25(86.2)$ & $4(13.8)$ & \\
\hline Invasive depth (T) & & & & 0.571 & & & 0.065 \\
\hline $\mathrm{T} 1 / 2$ & $5(22.7)$ & $6(27.3)$ & $11(50.0)$ & & $18(81.8)$ & $4(18.2)$ & \\
\hline T3/4 & $12(13.6)$ & $26(29.5)$ & $50(56.8)$ & & $89(94.7)$ & $5(5.3)$ & \\
\hline Lymph node metastasis (N) & & & & 0.142 & & & 0.299 \\
\hline NO & $8(17.8)$ & $17(37.8)$ & $20(44.4)$ & & $47(95.9)$ & $2(4.1)$ & \\
\hline N1 & $9(13.8)$ & $15(23.1)$ & $41(63.1)$ & & $60(89.6)$ & $7(10.4)$ & \\
\hline pTNM stage & & & & $0.080^{d}$ & & & 0.743 \\
\hline$\| A / I B$ & $9(18.0)$ & $19(38.0)$ & $22(44.0)$ & & $49(90.7)$ & $5(9.3)$ & \\
\hline III/IV & $8(13.3)$ & 13(21.7) & $39(65.0)$ & & $58(93.5)$ & $4(6.5)$ & \\
\hline $\begin{array}{l}\text { Data are presented as numbers, } \\
\text { a } 0 \text { of } 116 \text { cases, } 6 \text { had tumor ne } \\
\text { b } P \text { values among }-,+ \text { and }++ \\
\text { ' } P \text { values between }-/+ \text { and }++ \\
\text { d } P=0.027 \text { between }-/+ \text { and }++\end{array}$ & $\begin{array}{l}\text {, with percentage } \\
\text { ests without any } \\
\text { groups by } \chi^{2} \text { te } \\
\text { groups by } \chi^{2} \text { te } \\
++ \text { groups. }\end{array}$ & $\begin{array}{l}\text { arenthese } \\
\text { stromal }\end{array}$ & tissue microa & & & & \\
\hline
\end{tabular}


Table 3. The correlation between laminin- $5 \gamma_{2}$ chain expression and SPARC expression

\begin{tabular}{|c|c|c|c|c|c|c|}
\hline \multirow{2}{*}{$\begin{array}{l}\text { Laminin-5 } \gamma_{2} \\
\text { chain expression }\end{array}$} & \multicolumn{3}{|c|}{ SPARC expression in stromal fibroblasts ${ }^{\mathrm{a}}$} & \multicolumn{3}{|c|}{ SPARC expression in cancer cells ${ }^{b}$} \\
\hline & - & + & ++ & - & + & ++ \\
\hline- & $11(37.9)$ & $12(41.4)$ & $6(20.7)$ & $27(84.4)$ & $3(9.4)$ & $2(6.3)$ \\
\hline+ & $6(9.5)$ & $15(23.8)$ & $42(66.7)$ & $64(97.0)$ & $1(1.5)$ & $1(1.5)$ \\
\hline++ & $0(0)$ & $5(27.8)$ & $13(72.2)$ & $16(88.9)$ & $0(0)$ & $2(11.1)$ \\
\hline
\end{tabular}

Laminin-5 is a hetero-trimeric glycoprotein composed of $\alpha_{3}$ and $\beta_{3}$ chains with a unique $\gamma_{2}$ chain, which are products of LAMA3, LAMB3, and LAMC2 genes, respectively ${ }^{[1]}$. Laminin-5 $\gamma_{2}$ chain is highly expressed in some malignant carcinomas such as gastric cancer, tongue cancer, colon cancer, cervical cancer, and malignant melanoma. In addition, its high expression is associated with cancer invasion, metastasis, and prognosis $^{[25]}$.

Laminin-5 $\gamma_{2}$ chain was found localized at the basement membrane of normal esophageal mucosa and was expressed in the cytoplasm of cancer cells near the edge of esophageal SCC nests. Of 116 patients, 66 tested weakly positive and 18 tested strongly positive for laminin-5 $\gamma_{2}$ chain expression. The percentage of positive cells was more than $30 \%$ in strongly positive group. So $15.5 \%(18 / 116)$ of the cases were positive for laminin-5 $\gamma_{2}$ chain expression in more than $30 \%$ of the carcinoma cells. The positive ratio was slightly less than that reported by Yamamoto et al. ${ }^{[9]}$, in which $44 \%$ of the cases were positive for laminin-5 $\gamma_{2}$ chain expression in more than $30 \%$ of the carcinoma cells at the invasive front of esophageal SCC, possibly due to the differences in tissue sources, with tissue microarray employed in our study and traditional sections employed in their study. Most of tumors strongly expressing laminin-5 $\gamma_{2}$ chain grew in cords or small nests, exhibiting typical growing patterns in poorly differentiated SCC. In contrast, tumors weakly or not expressing laminin-5 $\gamma_{2}$ chain grew in large nests or large sheets, which are common in well-differentiated SCC. These observations are consistent with the conclusion that the expression of laminin-5 $\gamma_{2}$ chain was related to tumor differentiation.

It has been reported that the ability of cancer cell to migrate and invade is enhanced when laminin-5 $\gamma_{2}$ chain is cleaved by MMP, such as MMP-2 and/or MT1-MMP, secreted from cancer cells or adjacent stromal cells. Therefore, laminin-5 $\gamma_{2}$ chain may be an effective target during cell migration and invasion ${ }^{[11]}$. In addition, cytoplasmic accumulation of laminin-5 $\gamma_{2}$ chains may disrupt the synthesis of other laminin proteins, facilitating an increased capacity for cancer cell invasion ${ }^{[25]}$. Finally, studies have suggested overexpression of laminin-5 $\gamma_{2}$ chain increased cell proliferation, thereby promoting tumor growth ${ }^{[12]}$. In the present study, we observed the expression of laminin-5 $\gamma_{2}$ chain was not related to invasive depth, lymph node metastasis, or pTNM stage, but was an indicator of poor prognosis as revealed by survival analysis.

Matricellular proteins include a group of non-homologous molecules regulating the interaction of cells with the extracellular matrix. Matricellular proteins are different from extracelluar matrix proteins such as laminin due to lacking of participation in extracellular matrix formation. SPARC is representative of non-structural matricellular proteins and is of significant importance due to its enhanced expression in many cancers. Both cancer cells and stromal cells can express SPARC protein, with SPARC mRNA visualized in these cells by in situ hybridization ${ }^{[26]}$. In this study, cytoplasmic SPARC expression was only elevated in a few poorly differentiated esophageal SCC and immunostaining signals were generally localized in carcinoma cells at the invasive front. This expression pattern was very similar to that of laminin-5 $\gamma_{2}$ chain. In contrast, SPARC expression was elevated in the majority of stromal fibroblasts, displaying a strong profile along the negative background of cancer cells.

SPARC possibly influences tumor invasion and metastasis by altering the cytoskeletal structure, thereby affecting cellular adhesion. In addition, the expression of SPARC increases MMP expression in fibroblasts and monocytes. MMP facilitates the degradation of extracellular matrix components, leading to the increased ability of cancer cell movement related to cancer invasion, metastasis, and prognosis. In the present study, the expression level of SPARC in stromal fibroblasts had a tendency to correlate with PTNM stages. Survival analysis showed that high expression of stromal SPARC was associated with poor prognosis. This observation was consistent with earlier studies reporting a significant correlation between the elevated expression of SPARC mRNA and poor prognosis ${ }^{[10]}$.

The expression levels of laminin-5 $\gamma_{2}$ chain and SPARC were analyzed at different pTNM stages, which are representative of the progression of esophageal SCC. In patients with stage-IIA and -IIB esophageal 

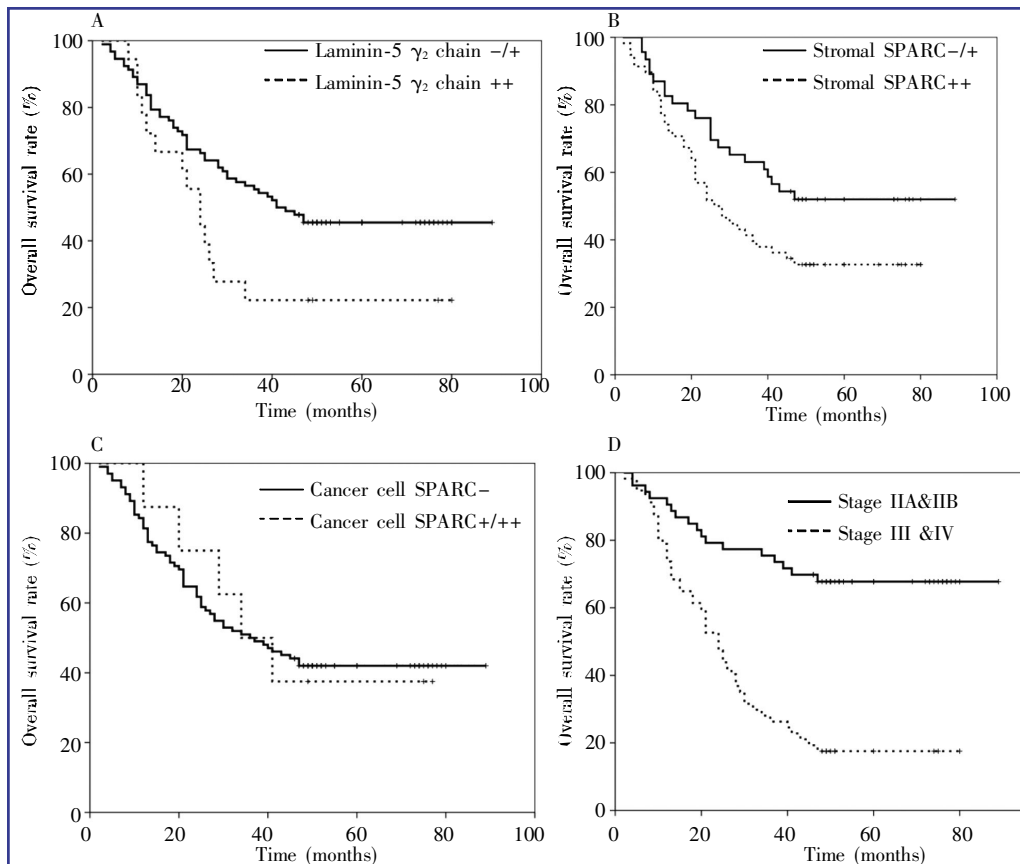

$$
\text { D }
$$
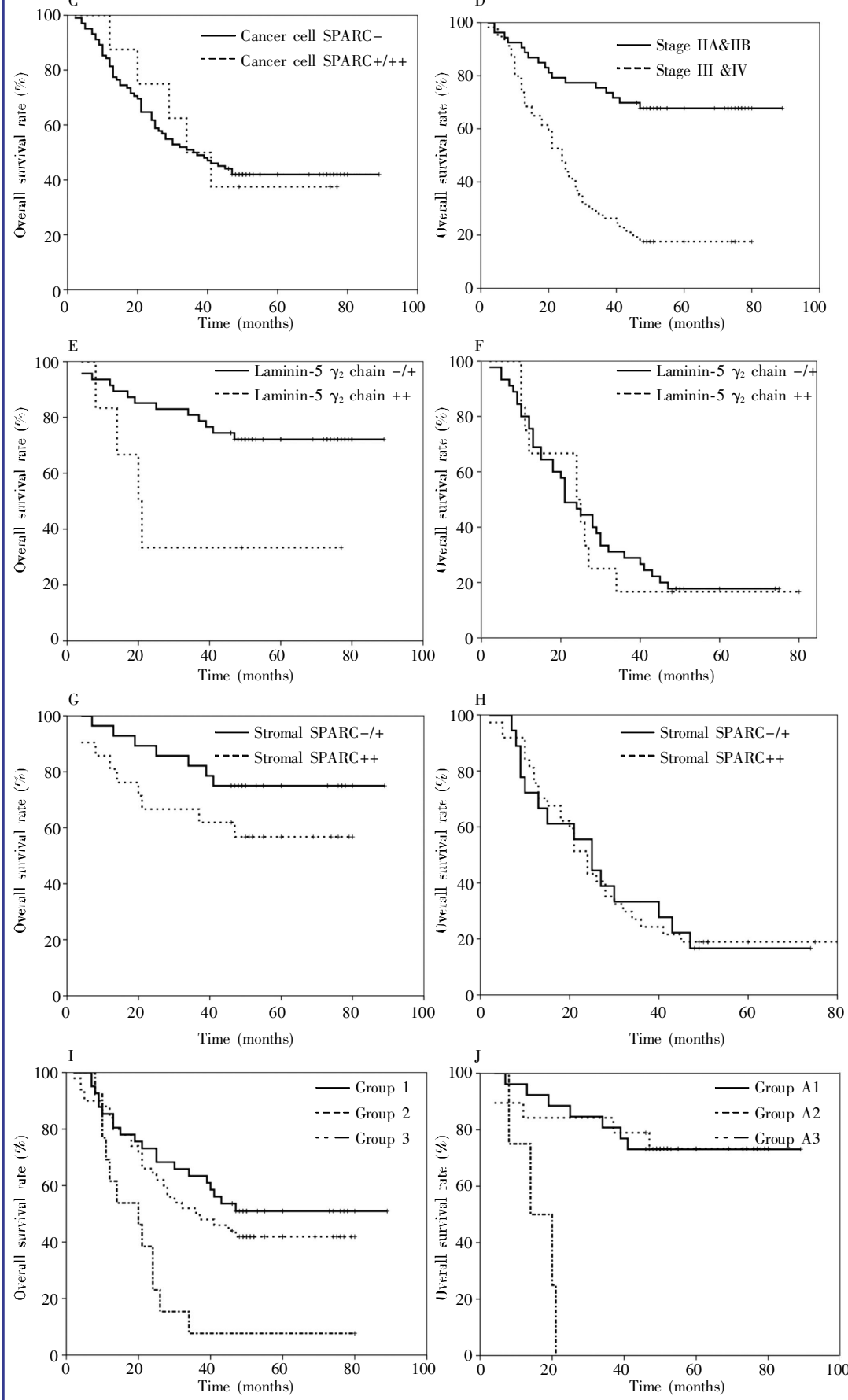

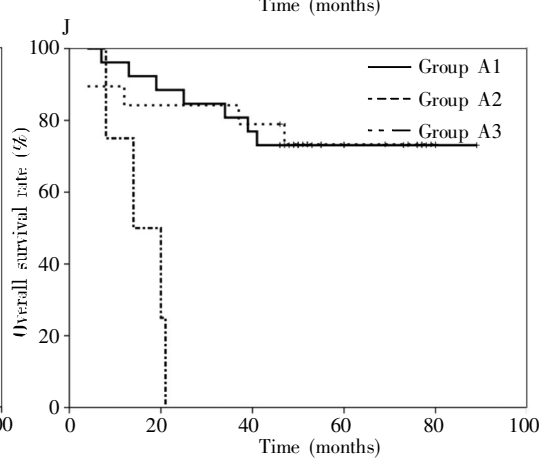

Figure 2. Survival curves of various groups of patients with esophageal SCC. A, patients with tumors strongly expressing (++) laminin-5 $\gamma_{2}$ chain had a significantly shorter overall survival time than did those with tumors not expressing $(-)$ and weakly expressing $(+)$ laminin-5 $\gamma_{2}$ chain $(P=0.032)$. B, patients with tumors strongly expressing (++) SPARC in stromal fibroblasts had a significantly shorter overall survival time than did those with tumors not expressing $(-)$ and weakly expressing $(+)$ SPARC in stromal fibroblasts $(P=$ $0.034)$. C, the overall survival curve of patients with tumor cells expressing SPARC $(+/++)$ in stromal fibroblasts was not statistically different from that of patients with tumor cells not expressing SPARC $(-)$ in stromal fibroblasts $(P=0.944)$. D, patients with stageIII/IV esophageal SCC had significantly shorter overall survival time than did those with stage-IIA/IIB diseases $(P<0.001)$. E, among patients with stage-IIA/IIB esophageal SCC, patients with tumors strongly expressing (++) laminin-5 $\gamma_{2}$ chain had significantly shorter overall survival time than did those with tumors not expressing (-) and weakly expressing (+) laminin-5 $\gamma_{2}$ chain $(P=0.023)$. F, among the patients with stageIII/IV esophageal SCC, the overall survival curve of patients with tumor cells strongly expressing (++) laminin-5 $\gamma_{2}$ chain was not statistically different from that of patients with tumor cells not expressing $(-)$ and weakly expressing $(+)$ laminin-5 $\gamma_{2}$ chain $(P=0.844)$. $G$, among patients with stage- IIA/IIB esophageal SCC, patients with tumors strongly expressing (++) SPARC in stromal fibroblasts had shorter overall survival time than did those with tumors not expressing $(-)$ and weakly expressing (+) SPARC in stromal fibroblasts, but without significant difference $(P=0.154)$. $\mathrm{H}$, among the patients with stage-III/IV esophageal SCC, the overall survival curve of patients with tumor cells strongly expressing (++) SPARC in stromal fibroblasts was not statistically different from that of patients with tumor cells not expressing $(-)$ and weakly expressing $(+)$ SPARC in stromal fibroblasts $(P=0.988)$. I, patients were classified into three groups, according to the expressions of laminin-5 $\gamma_{2}$ chain and/or SPARC in stromal fibroblasts. In group one, neither laminin-5 $\gamma_{2}$ chain nor SPARC is highly expressed; in group two, either laminin-5 $\gamma_{2}$ chain or SPARC is highly expressed; and in group three, both laminin-5 $\gamma_{2}$ chain and SPARC are highly expressed. Patients within group three had significantly shorter overall survival time than did patients in group one or group two $(P=0.001)$. J, patients with stage-IIA/IIB esophageal SCC in group A were further classified into three groups, according to the expressions of laminin-5 $\gamma_{2}$ chain and/or SPARC in stromal fibroblasts. Group A1 consists of cases in which neither laminin-5 $\gamma_{2}$ chain nor SPARC highly expressed, group A2 consists of cases in which either laminin-5 $\gamma_{2}$ chain or SPARC highly expressed, and group A3 consists of cases in which both laminin-5 $\gamma_{2}$ chain and SPARC highly expressed. Patients within group A3 had significantly shorter overall survival time than did patients in group A1 or group A2 $(P<0.001)$. 
SCC, the elevated expressions of laminin-5 $\gamma_{2}$ chain and SPARC were associated with poor survival, suggesting laminin-5 $\gamma_{2}$ chain and SPARC play important roles in stage-II esophageal SCC. Thus, simultaneous high expression of both laminin-5 $\gamma_{2}$ chain and SPARC is a strong prognostic indicator for survival of patients with stage-II disease.

Laminin-5 $\gamma_{2}$ chain and SPARC expressions were enhanced in esophageal SCC and predominantly expressed in cancer cells contacting the stroma at the edge of cancer nests. Although SPARC expression was mostly detected in the stroma, laminin-5 $\gamma_{2}$ chain expression was detectable in cancer cells located at the edge of cancer nests. It has been reported SPARC interacts with laminin and SPARC expression is related to laminin production ${ }^{[19-21]}$. However, the relationship between laminin-5 $\gamma_{2}$ chain expression and SPARC expression has not been investigated. We speculate overexpression of SPARC in esophageal SCC induces laminin-5 $\gamma_{2}$ chain production, providing a mechanism for
SPARC to facilitate the progress and metastasis of esophageal SCC. Further studies are warranted to confirm this hypothesis.

The elevated expressions of laminin-5 $\gamma_{2}$ chain and SPARC may be indicators of poor prognosis in esophageal SCC patients. As both laminin-5 $\gamma_{2}$ chain and SPARC function in stage-II esophageal SCC and reflect disease progression, detection of these two glycoproteins may be an indicator of clinical prognosis.

\section{Acknowledgment}

This work was supported by National Natural Science Foundation of the People's Republic of China (No. 30670964,30770973); Beijing Hope Run Special Fund (No. LC2007B51).

Received: 2010-02-20; revised: 2010-05-16; accepted: 2010-05-25.

\section{References}

[1] Burgeson RE, Chiquet $M$, Deutzmann $R$, et al. $A$ new nomenclature for the laminins [J]. Matrix Biol, 1994,14(3):209-211.

[2] Koukourakis MI, Giatromanolaki A, Brekken RA, et al. Enhanced expression of SPARC/osteonectin in the tumor-associated stroma of non-small cell lung cancer is correlated with markers of hypoxia/ acidity and with poor prognosis of patients [J]. Cancer Res, 2003, 63(17):5376-5380.

[3] Shinto E, Tsuda H, Ueno H, et al. Prognostic implication of laminin5 gamma 2 chain expression in the invasive front of colorectal cancers, disclosed by area-specific four-point tissue microarrays [J]. Lab Invest, 2005,85(2):257-266.

[4] Lenander C, Habermann JK, Ost A, et al. Laminin-5 gamma 2 chain expression correlates with unfavorable prognosis in colon carcinomas [J]. Anal Cell Pathol, 2001,22(4):201-209.

[5] Wang CS, Lin KH, Chen SL, et al. Overexpression of SPARC gene in human gastric carcinoma and its clinic-pathologic significance [J]. Br J Cancer, 2004,91(11):1924-1930.

[6] Zhao ZS, Wang YY, Chu YQ, et al. SPARC is associated with gastric cancer progression and poor survival of patients [J]. Clin Cancer Res, 2010,16(1):260-268.

[7] Zhang J, Zhang Q, Zhang Q, et al. Expression and clinical significance of SPARC in clinical stage II tongue squamous cell carcinoma [J]. Ai Zheng, 2009,28(1):68-71. [in Chinese]

[8] Kato $Y$, Nagashima $Y$, Baba $Y$, et al. Expression of SPARC in tongue carcinoma of stage II is associated with poor prognosis: an immunohistochemical study of 86 cases [J]. Int $\mathrm{J}$ Mol Med, 2005, 16(2):263-268.

[9] Yamamoto $\mathrm{H}$, Itoh $\mathrm{F}$, Iku S, et al. Expression of the gamma(2) chain of laminin-5 at the invasive front is associated with recurrence and poor prognosis in human esophageal squamous cell carcinoma [J]. Clin Cancer Res, 2001,7(4):896-900.

[10] Yamashita K, Upadhay S, Mimori K, et al. Clinical significance of secreted protein acidic and rich in cystein in esophageal carcinoma and its relation to carcinoma progression [J]. Cancer, 2003,97(10):2412-2419.

[11] Pyke C, Romer J, Kallunki P, et al. The gamma 2 chain of kalinin/ laminin 5 is preferentially expressed in invading malignant cells in human cancers [J]. Am J Pathol, 1994,145(4):782-791.

[12] Skyldberg B, Salo S, Eriksson E, et al. Laminin-5 as a marker of invasiveness in cervical lesions [J]. J Natl Cancer Inst, 1999,91 (21):1882-1887.

[13] Porte H, Chastre E, Prevot S, et al. Neoplastic progression of human colorectal cancer is associated with overexpression of the stromelysin-3 and BM-40/SPARC genes [J]. Int J Cancer, 1995,64 (1):70-75

[14] Tremble PM, Lane TF, Sage EH, et al. SPARC, a secreted protein associated with morphogenesis and tissue remodeling, induces expression of metalloproteinases in fibroblasts through a novel extracellular matrix-dependent pathway [J]. J Cell Biol, 1993,121 (6):1433-1444.

[15] Shankavaram UT, DeWitt DL, Funk SE, et al. Regulation of human monocyte matrix metalloproteinases by SPARC [J]. J Cell Physiol, 1997,173(3):327-334.

[16] Gilles C, Bassuk JA, Pulyaeva $H$, et al. SPARC/osteonectin induces matrix metalloproteinase 2 activation in human breast cancer cell lines [J]. Cancer Res, 1998,58(23):5529-5536.

[17] Rich JN, Shi Q, Hjelmeland M, et al. Bone-related genes expressed in advanced malignancies induce invasion and metastasis in a genetically defined human cancer model [J]. J Biol Chem, 2003,278(18): 15951-15957.

[18] Yamanaka M, Kanda K, Li NC, et al. Analysis of the gene expression of SPARC and its prognostic value for bladder cancer [J]. J Urol, 2001,166(6):2495-2499.

[19] Howe CC, Kath R, Mancianti ML, et al. Expression and structure of human SPARC transcripts: SPARC mRNA is expressed by human cells involved in extracellular matrix production and some of these cells show an unusual expression pattern [J]. Exp Cell Res, 1990, 188(2): 185-191.

[20] Yan Q, Perdue N, Blake D, et al. Absence of SPARC in murine lens epithelium leads to increased deposition of laminin-1 in lens capsule [J]. Invest Ophthalmol Vis Sci, 2005,46(12):4652-4660.

[21] Kamihagi K, Katayama M, Ouchi R, et al. Osteonectin/SPARC 
regulates cellular secretion rates of fibronectin and laminin extracellular matrix proteins [J]. Biochem Biophys Res Commun, 1994,200(1):423-428.

[22] Xue LY, Teng LH, Zou SM, et al. Expression of annexin I in different histological types of carcinomas [J]. Zhonghua Zhong Liu Za Zhi, 2007,29(6):444-448. [in Chinese]

[23] Xue LY, Ren LQ, Luo W, et al. Expression of Fas, Fas ligand, Fasassociated death domain protein, caspase 8 and mutant P53 protein in esophageal squamous cell carcinoma [J]. Zhonghua $Y_{i}$ Xue Za Zhi, 2007,87(3):150-154. [in Chinese]

[24] Moriya Y, Niki T, Yamada T, et al. Increased expression of laminin-
5 and its prognostic significance in lung adenocarcinomas of small size. An immunohistochemical analysis of 102 cases [J]. Cancer, 2001,91(6):1129-1141.

[25] Koshikawa N, Moriyama K, Takamura H, et al. Overexpression of laminin gamma2 chain monomer in invading gastric carcinoma cells [J]. Cancer Res, 1999,59(21):5596-5601.

[26] Wewer UM, Albrechtsen R, Fisher LW, et al. Osteonectin/SPARC/ $\mathrm{BM}-40$ in human decidua and carcinoma, tissues characterized by de novo formation of basement membrane [J]. Am J Pathol, 1988, 132(2):345-355.
Submit your next manuscript to Chinese Journal of Cancer and take full advantage of:

- Open access

- No charge to authors

- Quickly published

- Thorough peer review

- Professionally edited

- No space constraints

- Indexed by PubMed, CA, and Google Scholar

Submit your manuscript at www.cjcsysu.com 